\title{
Penifulvins B-E and a New Silphinene Analogue: Sesquiterpenoids from a Fungicolous Isolate of Penicillium griseofulvum
}

\author{
Sang Hee Shim, ${ }^{\dagger}$ James B. Gloer, ${ }^{*}{ }^{\dagger}$ and Donald T. Wicklow ${ }^{\ddagger}$ \\ ${ }^{\dagger}$ Department of Chemistry, University of Iowa, Iowa City, Iowa 52242 and \\ ${ }^{*}$ Mycotoxin Research Unit, Agricultural Research Service, National Center for \\ Agricultural Utilization Research, USDA, Peoria, Illinois 61604
}

\section{List of Supporting Information}

Figure S1. ${ }^{1} \mathrm{H}$ NMR spectrum of penifulvin B $\left(\mathbf{2} ; 400 \mathrm{MHz}, \mathrm{CDCl}_{3}\right)$

Figure S2. ${ }^{13} \mathrm{C}$ NMR spectrum of penifulvin $\mathrm{B}\left(\mathbf{2} ; 100 \mathrm{MHz}, \mathrm{CDCl}_{3}\right)$

Figure S3. ${ }^{1} \mathrm{H}$ NMR spectrum of penifulvin C (3; $\left.400 \mathrm{MHz}, \mathrm{CDCl}_{3}\right)$

Figure S4. ${ }^{13} \mathrm{C}$ NMR spectrum of penifulvin $\mathrm{C}\left(\mathbf{3} ; 100 \mathrm{MHz}, \mathrm{CDCl}_{3}\right)$

Figure S5. ${ }^{1} \mathrm{H}$ NMR spectrum of penifulvin D (4; $\left.400 \mathrm{MHz}, \mathrm{CDCl}_{3}\right)$

Figure S6. ${ }^{13} \mathrm{C}$ NMR spectrum of penifulvin $\mathrm{D}\left(\mathbf{4} ; 100 \mathrm{MHz}, \mathrm{CDCl}_{3}\right)$

Figure S7. ${ }^{1} \mathrm{H}$ NMR spectrum of penifulvin E (5; $\left.400 \mathrm{MHz}, \mathrm{CDCl}_{3}\right)$

Figure S8. ${ }^{13} \mathrm{C}$ NMR spectrum of penifulvin $\mathrm{E}\left(\mathbf{5} ; 100 \mathrm{MHz}, \mathrm{CDCl}_{3}\right)$

Figure S9. ${ }^{1} \mathrm{H}$ NMR spectrum of 12-hydroxysilphinene 15-oic acid (6; $\left.400 \mathrm{MHz}, \mathrm{CDCl}_{3}\right)$

Figure S10. ${ }^{13} \mathrm{C}$ NMR spectrum of 12-hydroxysilphinene 15-oic acid (6; $100 \mathrm{MHz}$, $\left.\mathrm{CDCl}_{3}\right)$ 


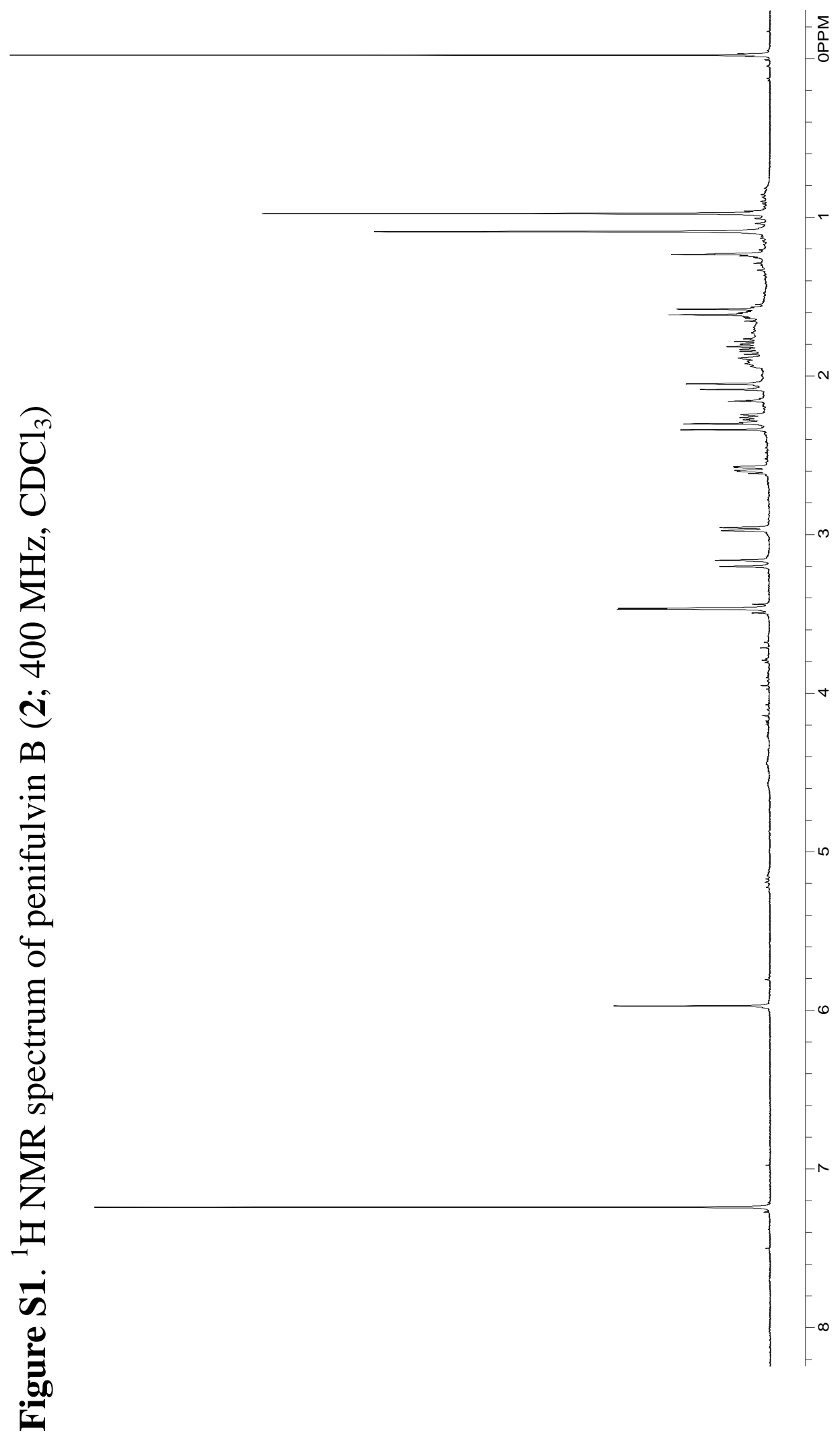




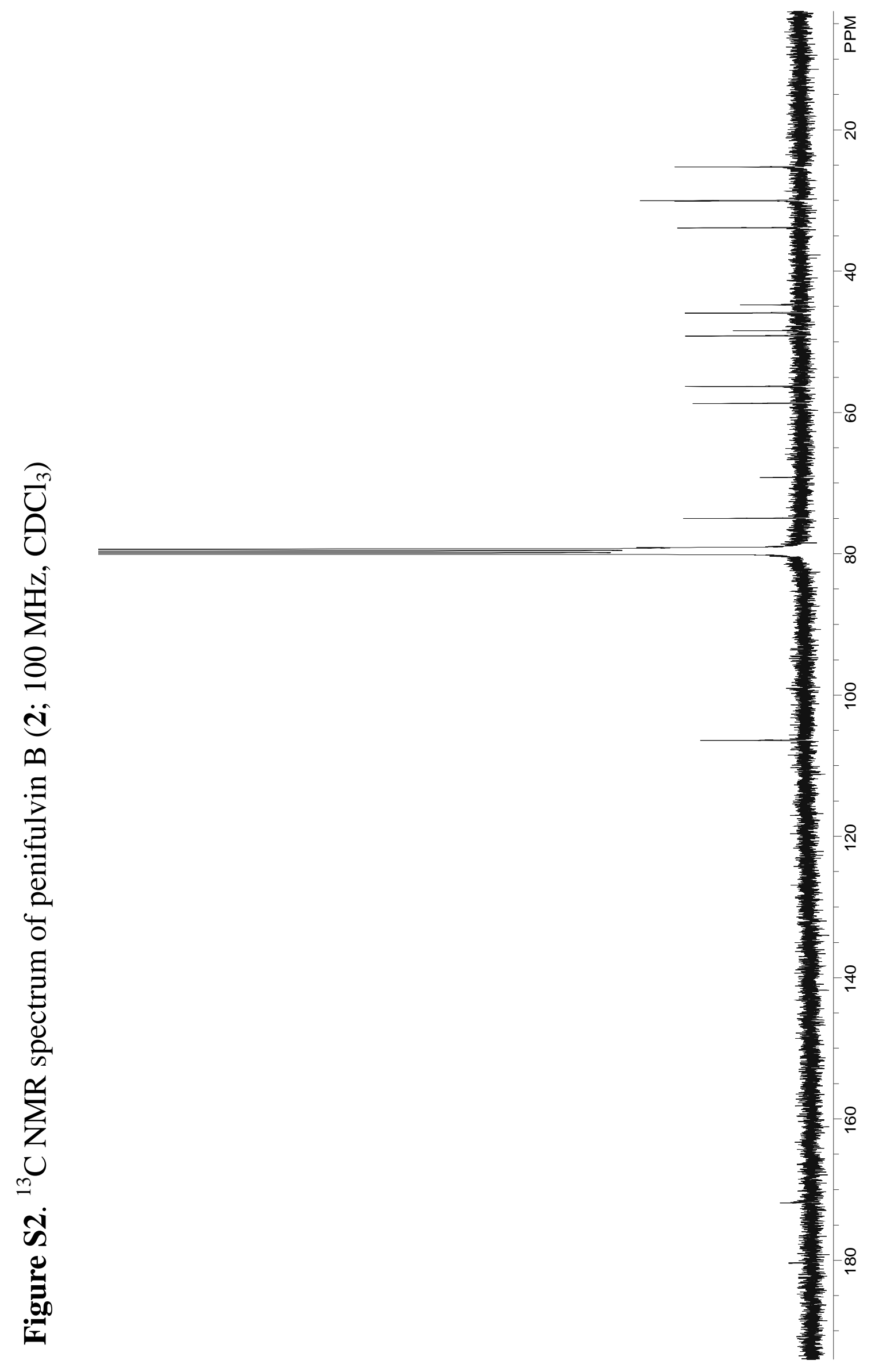




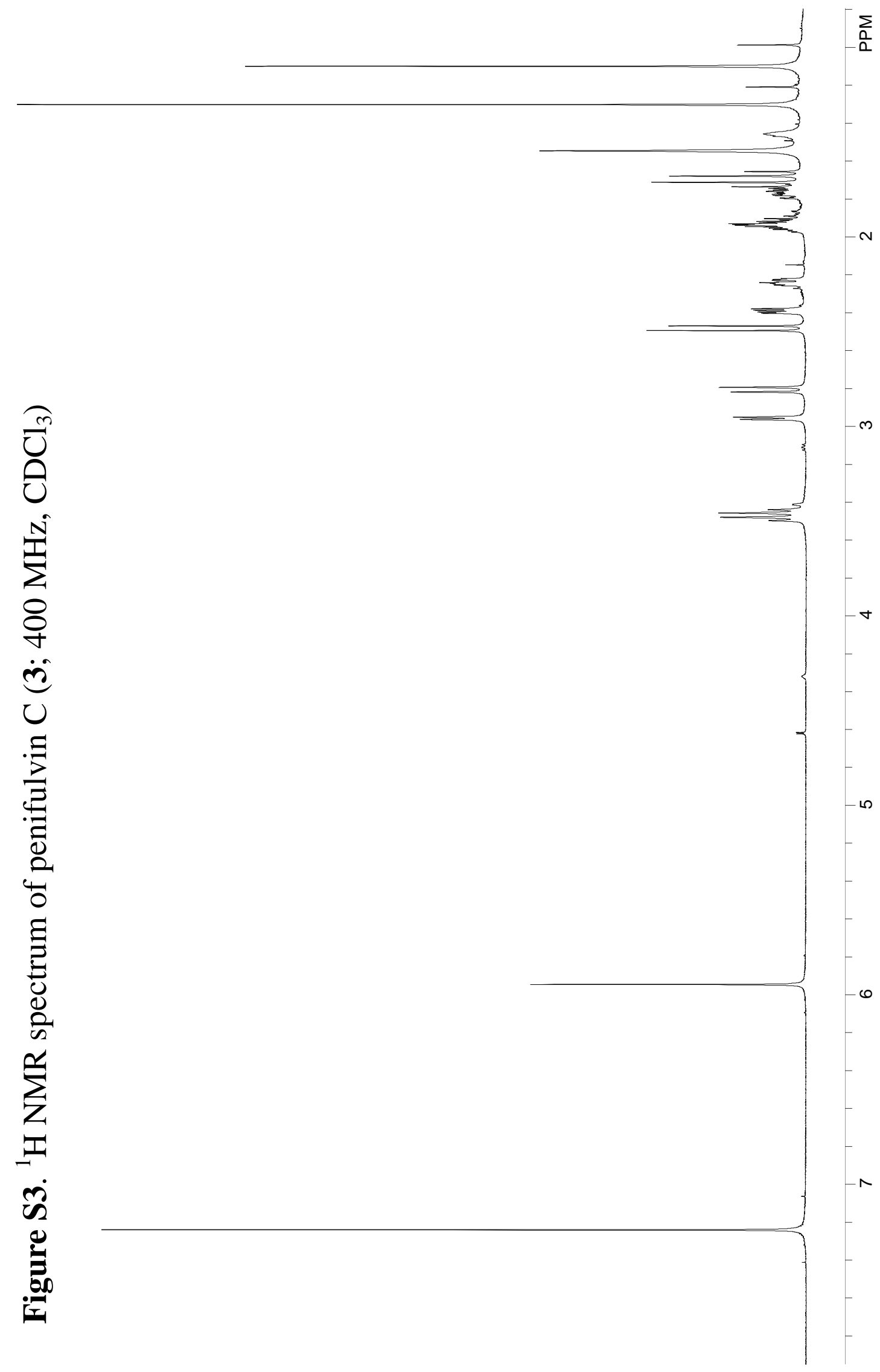




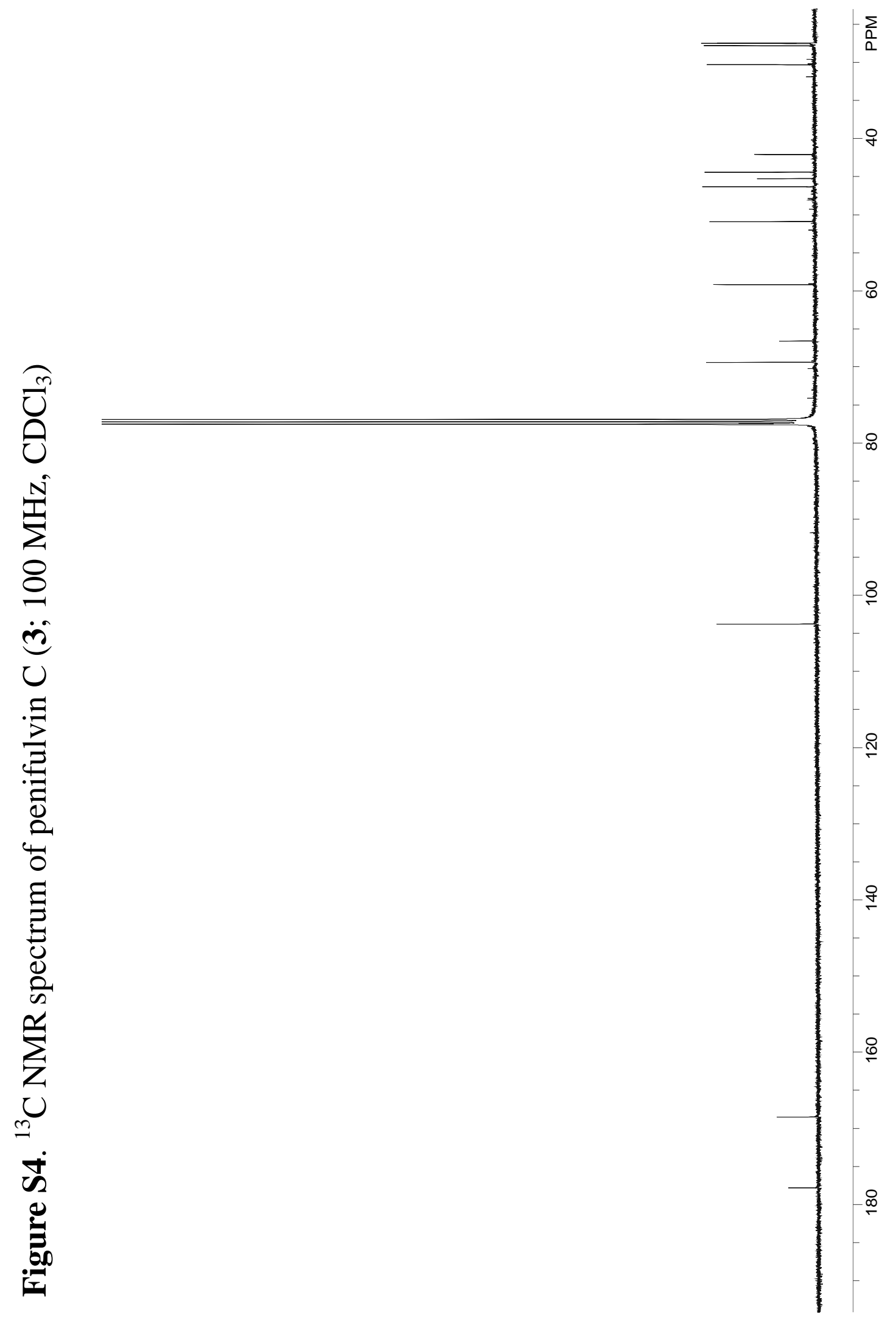




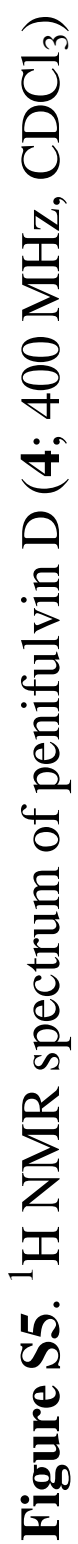

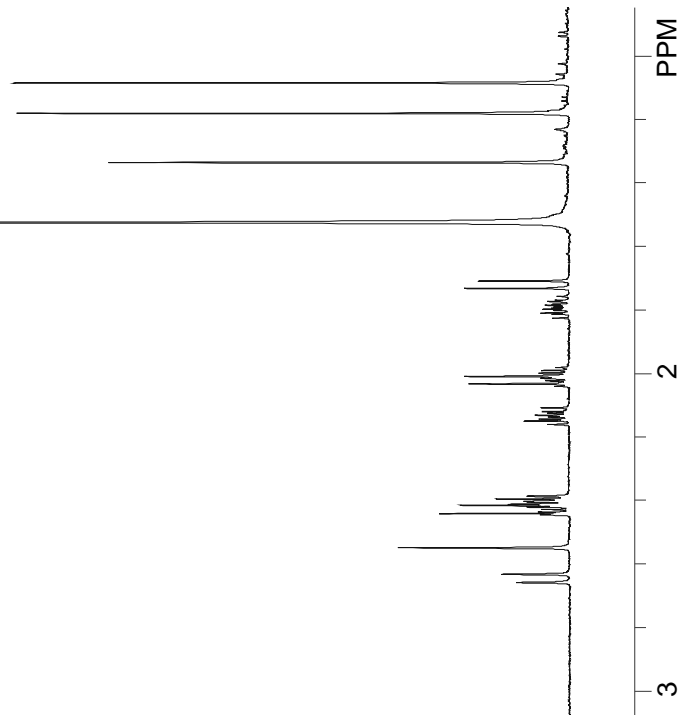

$-m$ 


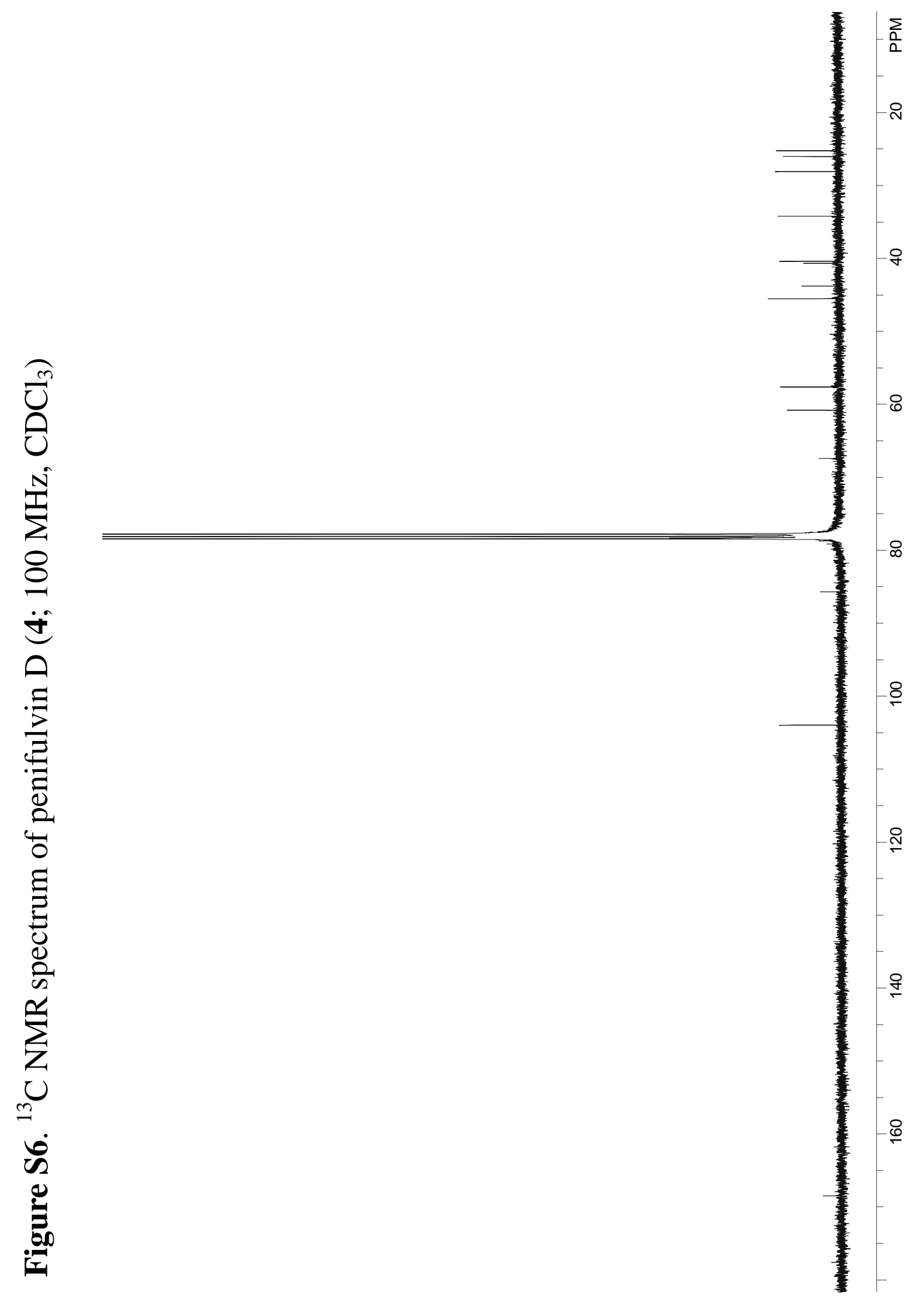




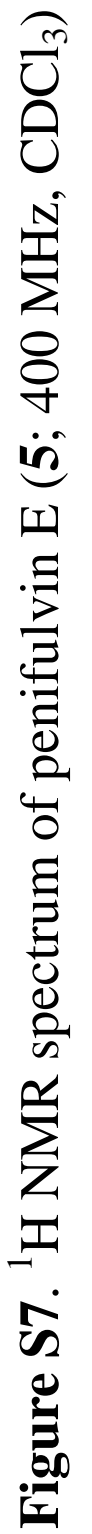




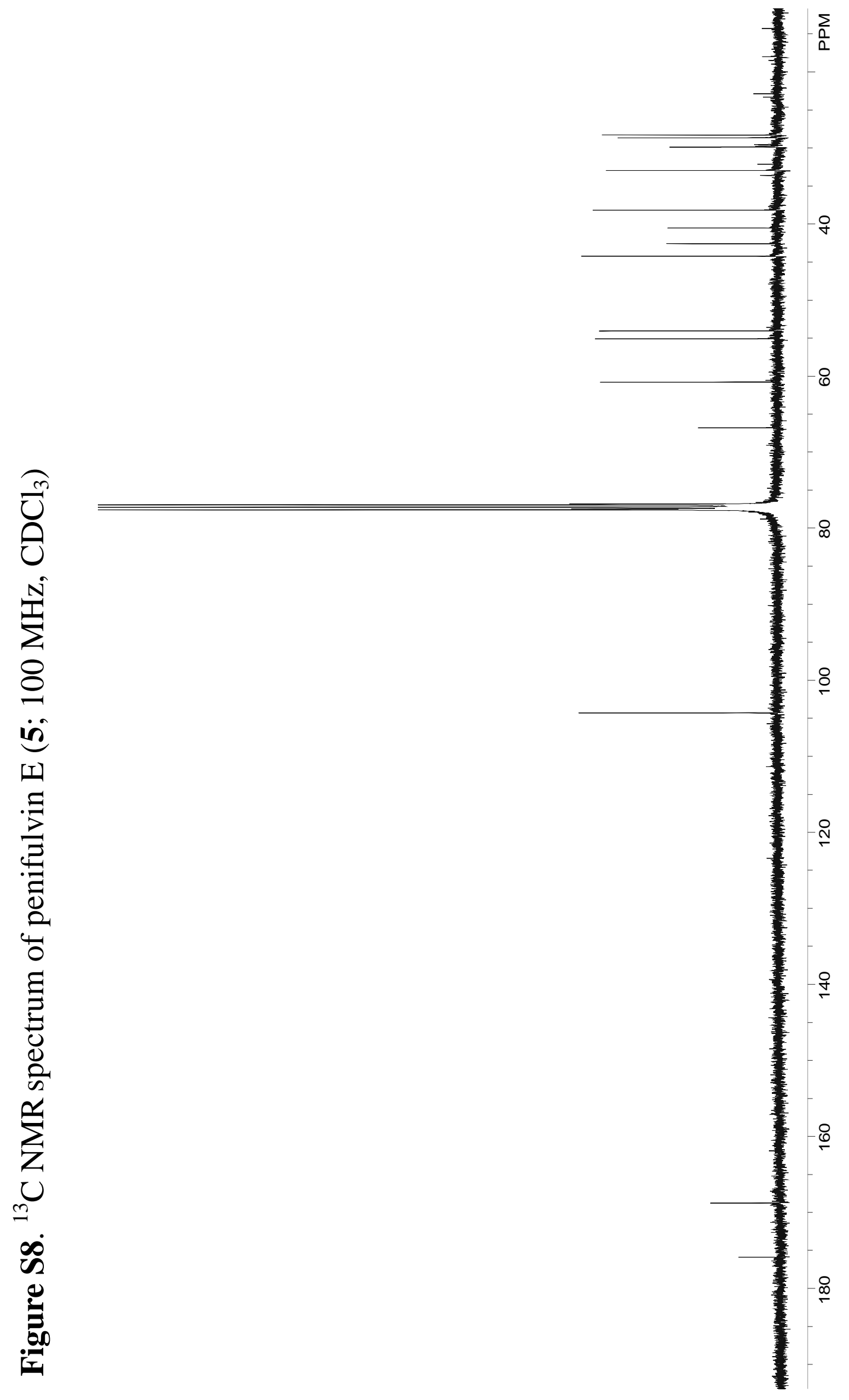



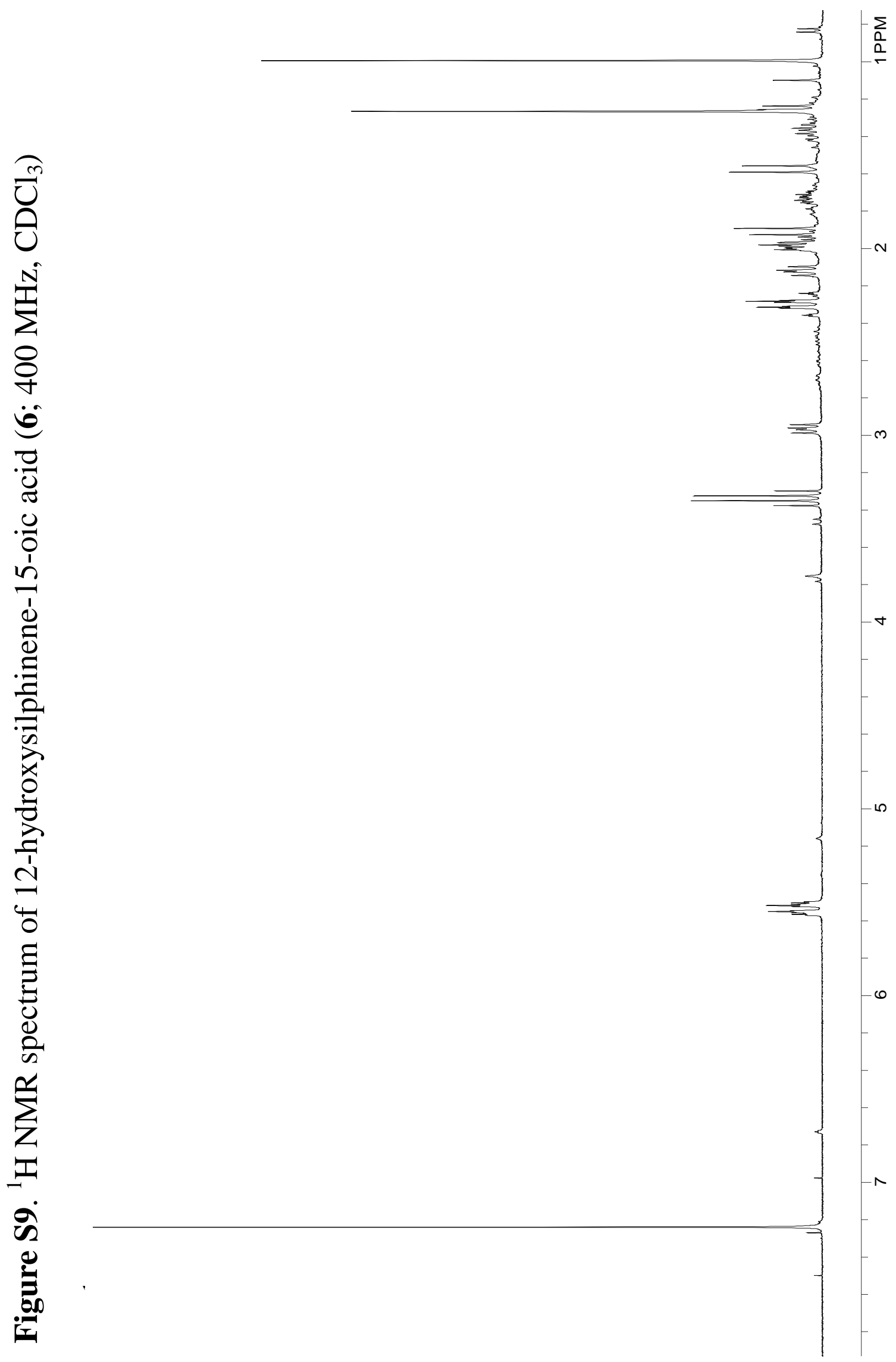


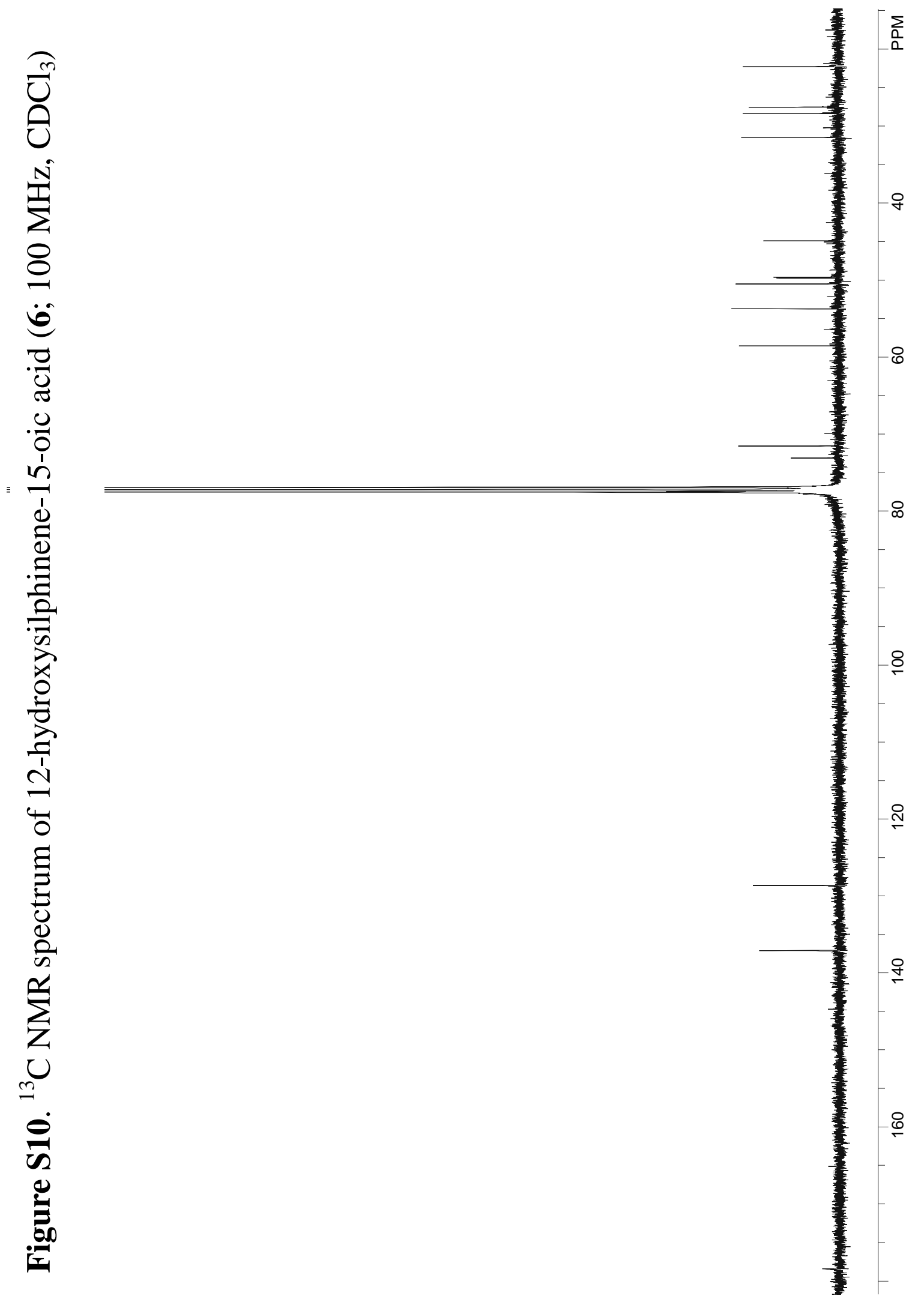

